\title{
A Portable Neutron/Tunable X-Ray Source Based on Inertial Electrostatic Confinement
}

\author{
George H. Miley \\ University of Illinois, Fusion Studies Laboratory \\ 102 NEL, 103 S. Goodwin Avenue \\ Urbana, IL 61801, U.S.A.
}

Ph: 217-333-3772, Fax: 217-333-2906, email: g-miley@uiuc.edu

\begin{abstract}
Inertial Electrostatic Confinement (IEC) offers a unique ion-beam-plasma-target configuration for production of neutrons via D-D or D-T fusion reactions. Research at the U. of IL has developed a unique "STAR" mode of operation where a basketball-shaped grid in the spherical $(\mathrm{r} \sim 15 \mathrm{~cm})$ vacuum vessel creates intense ion beams focused at the center of the vessel, forming a dense fusing plasma core (target). Key advantages of this unique design are that grid sputtering is greatly reduced and good beam focusing is achieved. Commercial versions of this concept have been developed that offer $10^{7} 2.45-\mathrm{MeV}$ D-D neutrons $/ \mathrm{sec}$ (or $10^{9} / \mathrm{sec}$ D-T). Such units are typically used to replace Cf-252 sources for industrial NAA. Next generation devices with rates above $10 \% / \mathrm{sec}$ D-D are currently under development. The IEC also provides a small tunable $\mathrm{x}$-ray source $(5-100 \mathrm{keV})$ for research applications by reversing the grid potential and also installing electron emitters. The changeover requires several hours down time, or, if needed, a separate dedicated IEC $\mathrm{x}$-ray unit could be constructed.
\end{abstract}

\section{INTRODUCTION}

The U. of IL IEC uses a transparent cathode grid inside a vacuum vessel (anode) in a spherical configuration or a hollow cathode-anode


FIGURE 1 Photograph of a spherical unit in operation (above). The grid and dense core region, just visible in the port opening, are enlarged in the top right photograph. In the photograph of an operating cylindrical IEC (below, right), the dark ring structures are the electrodes

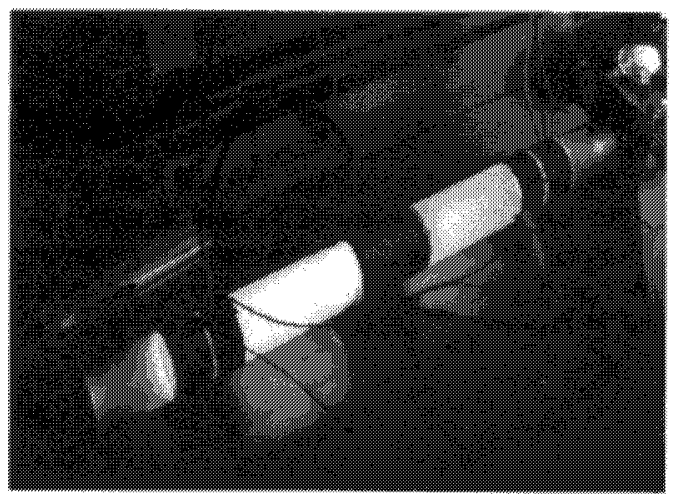

CP576, Application of Accelerators in Research and Industry - Sixteenth Int' 'l. Conf., edited by J. L. Duggan and I. L. Morgan (C) 2001 American Institute of Physics 0-7354-0015-6/01/\$18.00 
configuration in cylindrical geometry $[1,2]$. These two geometries are illustrated in the photographs in Fig. 1. Their construction is illustrated in Fig. 2. The current spherical unit employs a single grid for simplicity, although multigrid versions offer somewhat better efficiency. Likewise, the cylindrical unit is based on the simplest electrode design and arrangement for ease of construction.

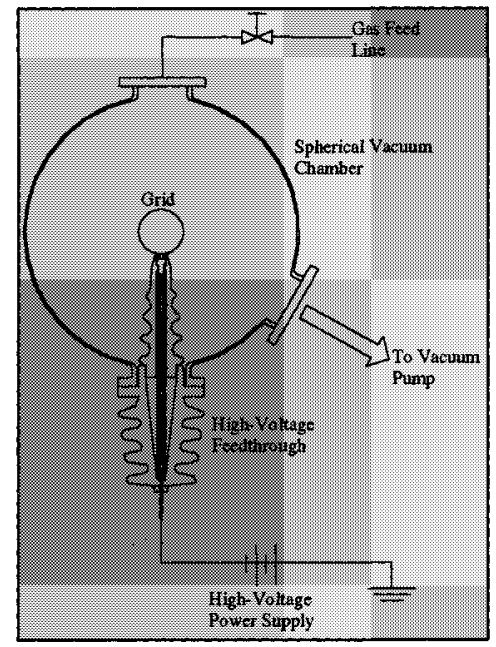

(a)

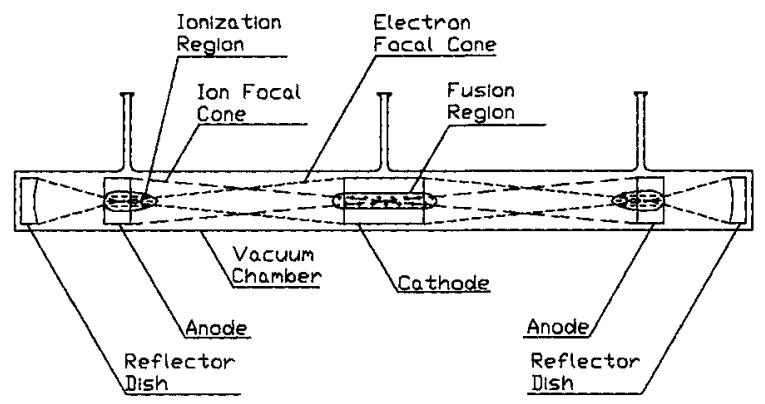

(b)

FIGURE 2. Schematic of the cross section of (a) a spherical IEC unit showing the grid and high voltage insulator structure and (b) a sketch of the cylindrical device and electrode structure.
Typical operating parameters are given in Table 1. A wide range of geometric dimensions are possible. However, once the electrode spacing is selected, the voltage and pressure are fixed by the Paschen breakdown relation [3].

TABLE 1. Typical Operational Parameters

\begin{tabular}{|l|l|}
\hline Parameter & Value \\
\hline Pressure & $0.1-5.0 \mathrm{~m}$ Torr \\
\hline Voltage & $50-100 \mathrm{kV}$ \\
\hline Device Diameter & $50-100 \mathrm{~cm}$ \\
\hline Input Power & $0.1-10 \mathrm{~kW}$ \\
\hline
\end{tabular}

The spherical unit offers a "point-type" source with an off-the-shelf steady-state rate of $10^{7} 2.5-\mathrm{MeV} \mathrm{D}$ D neutrons $/ \mathrm{sec}(\mathrm{n} / \mathrm{s})$ and possibly up to $10^{8} \mathrm{D}-\mathrm{D} \mathrm{n} / \mathrm{s}$ in $50-\mathrm{Hz}$ pulsed versions. (With a D-T gas fill, these rates would increase by the cross-section ratio of about $10^{2}$ ). The cylindrical version offers similar neutron rates, but provides a "line-like" source extending up to $-25 \mathrm{~cm}$ length. The latter configuration is most advantageous for applications requiring uniform coverage of broad surface areas, e.g. analysis of materials carried on a conveyor belt. Versions of the spherical unit are now produced commercially by Daimler-Benz Aerospace Corporation (DASA) [4].

\section{TUNABLE X-RAY SOURCE}

Another important advantage of the IEC, especially the smaller laboratory units, is that it can be converted to an attractive tunable $\mathrm{x}$-ray source with minimum alteration of the apparatus [5]. X-ray operation essentially involves reversing electrode polarities and adding electron emitters.

The resulting electron Bremmstrahlung radiation has a broad energy spectrum extending up to the applied voltage $(\sim 10-60 \mathrm{kV})$. A typical measurement corresponding to an applied voltage of $30 \mathrm{kV}$ is shown in Fig. 3. A FWHM of about $15 \mathrm{kV}$ is obtained with a peak intensity at about $20 \mathrm{kV}$. This makes possible some small-scale laboratory $\mathrm{x}$-ray experiments that would otherwise necessitate traveling to a synchrotron-type "light' source. 


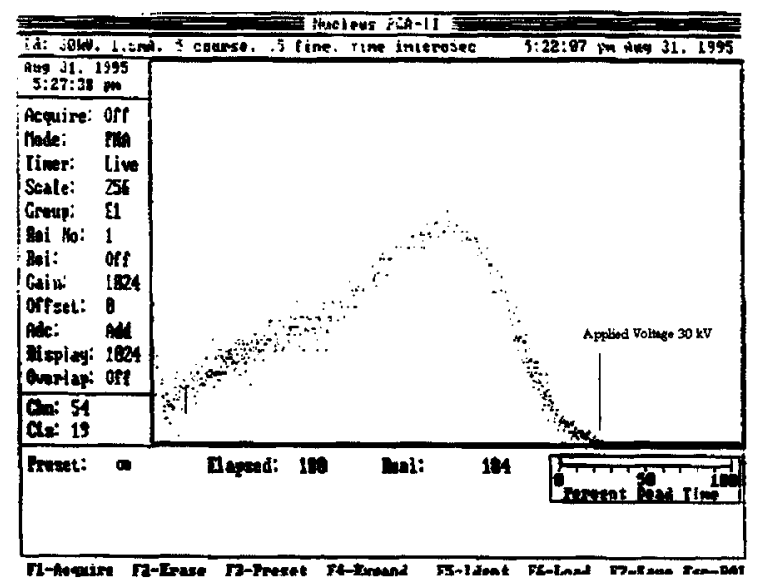

Figure 3. Measured X-ray intensity energy spectrum. The $y$-axis shows the normalized intensity. The peak energy occurs at $\sim 20 \mathrm{kV}$ ( $\mathrm{x}$-axis)

\section{RECENT PULSED OPERATION STUDIES}

Pulsed operation is attractive for certain NAA applications and also provides an attractive route to higher neutron yields since neutron production scales with ion current which can be very high using pulsed power technology [8]. The specialized pulser designed for such operation resembles a transmission line-type pulser. A hydrogen-thyratron switch is used to initiate the pulse and discharge the energy storage capacitors. Typical pulse lengths with this set-up are $\sim 10 \mu \mathrm{s}$, consistent with requirements set by the ion recirculation time in the potential well.

Neutron production as a function of pulsed current for several different cathode-grid designs is presented in Figure 4. Data was collected for pulsed operation for cathode voltages of $\sim 50 \mathrm{kV}$, and currents up to $17 \mathrm{~A}$.

The data in Figure 4 was taken with two different grids. The first, a small reference grid was used (Grid A); a second, larger grid was also used (Grid B). Grid B produced a slightly higher fusion reaction rate. This can be explained since the larger grid allows a lower neutral background pressure cf. Paschen theory of breakdown [3], reducing charge exchange losses. In conclusion, these preliminary results suggest pulsed operation of the IEC can be very attractive and provides an important compliment to conventional steady-state operation.

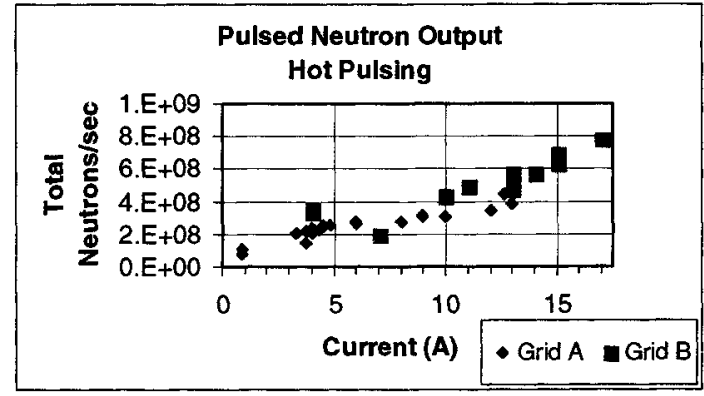

FIGURE 4. Pulsed neutron output at peak of pulse vs. peak cathode current.

\section{SCALE-UP ISSUES}

An understanding of the underlying plasmaelectrodynamic physics of the IEC is needed to evaluate its potential for scale up to even higher neutron rates. The spherical version will be used here to explain the basic phenomena. In it, the cathode grid extracts and accelerates ions created in a plasma discharge created between it and the anode (vacuum vessel wall). As these ions converge in a small volume around the center of the sphere, a virtual anode is created which accelerates and focuses electrons into a yet smaller concentric volume.

These converging electrons develop a negative

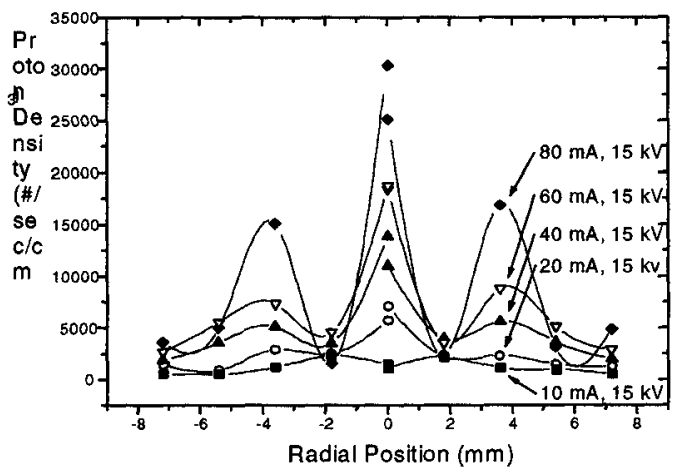

FIGURE 5. Measurement of the potential-well structure using a collimated D-D proton detector. 
potential well within the positive "hill," which traps ions, greatly reducing energetic ion leakage and creating an intense region of beam-beam fusion reactions. Y. Gu recently achieved a measurement of this potential structure using a collimated proton detector to observe the source rate profile of D-D protons across the central core region [9]. Some of his results are shown in Figure 5. A triple peak profile, indicative of a potential "double well", occurs at higher currents $(\geq 40 \mathrm{~ms})$. The ability to scale-up to yet higher neutron rates requires generating a larger and deeper ion trap, which in turn involves controlling ion energies and angular momentum while increasing ion currents. Since such scale-up involves a velocity-space (vs. physical space) phenomena, higher neutron rates do not force larger unit sizes. However, a modest size increase of the total system dimensions is required to accommodate voltage holding, increased cooling and radiation shielding requirements.

\section{CONCLUSIONS}

The IEC devices described here represent a new class of neutron sources based on ion-beam-plasmatarget fusion. They create a dense fusing region by focusing or concentrating the beam ions. In addition, energy efficiency is achieved by generation of a potential structure such that ions are trapped and recirculated. In contrast to conventional magnetic confinement fusion experimental devices, the IEC is unique in using electrostatic fields alone without the complication of magnetic field coils. The IEC uses ion inertia to obtain confinement without violating limits imposed by Earnshaw's theorem [10]. Key benefits of this approach are reflected in present devices through simplicity, compactness and long lifetime. Since the devices described here represent "first generation" designs, further improvements can be expected as research progresses.

\section{ACKNOWLEDGMENTS}

The IEC group at the U. of IL, Robert Stubbers, Brian Jurczyk, Mike Williams, and John DeMora, have been instrumental in this work. Helpful discussions with R. Nebel and D. Barnes (Los Alamos National Laboratory), R.W. Bussard ( $\left.\mathrm{MC}^{2}\right)$, M. Ohnishi (Kansai University), and R. Hirsch (Rand Corp), are gratefully acknowledged. This work was partially supported by DASA Contract No. 505013D MIL.

\section{REFERENCES}

[1] Miley, G. H. "A Portable Neutron/Tunable X-Ray Source Based on Inertial Electrostatic Confinement," Proceedings, Ninth Symposium on Radiation Measurements and Application, edited by H.C. Griffin, W.L. Rogers, and K. Rengan, North Holland, 1999.

[2] Miley, G. H. "A Novel 2.5 MeV D-D Neutron Source," Journal of Brachytherapy International, Vol. 1, No. 1, 111-121, (1997).

[3] Miley, G. H. et al., "Discharge Characteristics of the Spherical Inertial Electrostatic Confinement (IEC) Device," IEEE Trans. on Plasma Science, Vol 25, No. 4, pp. 733-739, August 1997.

[4] Miley, G. H. and Sved, J."The IEC Star-Mode Fusion Neutron Source for NAA-Status and Next-Step Designs", Proceedings, IRRMA '99, Raleigh, NC, 1999.

[5] Gu, Y. and Miley, G. H. "Spherical IEC Device as a Tunable X-ray Source," Bult. APS, 11, 1851 (1995).

[6] Gu, Y., Heck, P., and Miley, G. H. "Ion Focus Via Microchannels in Spherical Inertial Electrostatic Confinement and Its Pulsed Experimental Results," 1995 IEEE International Conference on Plasma Science, IEEE Conf. Rec. 95CH35796, 266-267 (1995).

[7] Jurczyk, B., Gu, Y., and Miley, G. H. "Resonant Ion Driven Oscillation (RIDO) Concept," Proceedings $39^{\text {th }}$ Annual Meeting Division of Plasma Physics, Pittsburgh, PA, Vol. 42, No. 10, p. 1818, November 17-21, 1997.

[8] Miley, G. H., Nadler, J. et al. "Issues for Development of Inertial Electrostatic Confinement (IEC) for Future Fusion Propulsion", $35^{\text {th }}$ AIAA/ASME/SAE/ASEE Joint Propulsion Conference, Los Angeles, CA, 1999, AIAA-99-2140.

[9] Gu, Y. and Miley, G. H. "Experimental Study of Potential Structure in a Spherical IEC Fusion Device," IEEE Transaction of Plasma Science, Vol 28, No. 1, 331-346, February 2000.

[10] Glasstone, S. \& Lovberg, R., Controlled Thermonuclear Reactions: An Introduction to Theory and Experiment, D. Van Nostrand Co., NY, 1960, p.47. 